\title{
Analysis Technology of Tennis Sports Match Based on Data Mining and Image Feature Retrieval
}

\author{
Hong Huang and Risheng Deng $\mathbb{D}$ \\ School of Sports Science, Lingnan Normal University, Zhanjiang, Guangdong 524048, China \\ Correspondence should be addressed to Risheng Deng; 2013141083016@stu.scu.edu.cn
}

Received 3 September 2020; Revised 25 September 2020; Accepted 30 September 2020; Published 14 October 2020

Academic Editor: Chuan Lin

Copyright (c) 2020 Hong Huang and Risheng Deng. This is an open access article distributed under the Creative Commons Attribution License, which permits unrestricted use, distribution, and reproduction in any medium, provided the original work is properly cited.

\begin{abstract}
Tennis game technical analysis is affected by factors such as complex background and on-site noise, which will lead to certain deviations in the results, and it is difficult to obtain scientific and effective tennis technical training strategies through a few game videos. In order to improve the performance of tennis game technical analysis, based on machine learning algorithms, this paper combines image analysis to identify athletes' movement characteristics and image feature recognition processing with image recognition technology, realizes real-time tracking of athletes' dynamic characteristics, and records technical characteristics. Moreover, this paper combines data mining technology to obtain effective data from massive video and image data, uses mathematical statistics and data mining technology for data processing, and scientifically analyzes tennis game technology with the support of ergonomics. In addition, this paper designs a controlled experiment to verify the technical analysis effect of the tennis match and the performance of the model itself. The research results show that the model constructed in this paper has certain practical effects and can be applied to actual competitions.
\end{abstract}

\section{Introduction}

Compared with traditional, technical, and tactical analysis methods, data mining technology can more clearly describe and analyze the process and sequence of each hitting technique, such as the position and route of the shot, and the process and sequence of winning and losing points. Moreover, it can help coaches to better grasp the commonalities and characteristics of players' skills and tactics, and provide reference and basis for the use of skills and tactics in future training and games [1]. In recent years, with the increase of opportunities for domestic players to participate in the competition, the increasingly abundant game videos have made the accumulation of technical and tactical data in the game continue to increase. How to explore the technical and tactical characteristics of excellent players from the rich data and provide a basis for the scientific decision-making of coaches and athletes has become one of the key and urgent problems in tennis theoretical research. In addition, the use of data mining technology to analyze the technical and tactical characteristics and laws of athletes in the game can obtain and find more valuable information and laws in a timely and accurate manner to make up for the deficiencies of traditional statistical methods. Therefore, it is necessary for tennis players to use data mining technology to assist them in making technical and tactical decisions [2].

Compared with the popularity of established sports such as football and basketball, tennis has relatively few followers. However, with the development of mass sports and the influence of international tennis, tennis has developed rapidly in my country in recent years. Moreover, more and more people have begun to pay attention to large-scale tennis matches, and the annual four Grand Slam matches and various masters have attracted the attention of many fans [3]. The Tennis Open has introduced "Eagle Eye" technology, which can accurately determine whether the ball is out of bounds. Players have two applications for the right to use Hawkeye in each set. After the referee accepts the application, they will broadcast the "Instant Replay" on the big screen to present the results of Hawkeye's calculation. The system takes no more than 10 seconds from data collection to result presentation. However, the system is 
composed of 8 or 10 high-speed cameras, four computers and a large screen, which makes the cost very high, and the amount of calculations when processing the collected data is very large [4]. Based on this, this article uses big data technology to analyze tennis sports matches, combined with machine vision to identify sports scenes, and improve the analysis effect of tennis sports matches.

\section{Related Work}

The literature [5] pointed out that when analyzing players and correcting their movements, a correct understanding of biomechanics will help coaches avoid focusing on peculiar, seemingly uncomfortable shots and help them focus on the timing of shots. The literature [6] proposed that the sequence of tennis coordination chain body segments is from the foot, hip, upper body, arm or shoulder, and elbow to wrist, and biomechanics is from knee flexion, hip rotation, body rotation, arm around shoulder, elbow extension, and forearm internal rotation to wrist rotation. The literature [7] analyzed the throw technique of tennis players in the Atlanta Olympic Games and showed that the throwing position is in front of the front foot or left front. High-speed camera technology is used for player analysis. The results show that many excellent players start to hit the ball after the ball has started to fall $(25-20 \mathrm{~cm})$.

The literature [8] mentioned technology alone cannot win the game; even if the technology is improved in daily training, if it cannot be played in the actual game, it is meaningless. At the same time, it is also mentioned that tennis is essentially control and anticontrol, and if the athlete controls the opponent throughout the game, he will definitely be able to win the game. Moreover, it is not only superlevel performance that can succeed. If the opponent can barely deal with it, the game will inevitably develop towards the side that is beneficial to oneself, and then victory can be expected. The literature [9] mentioned that in today's women's games, offense after serving is a very common tactic. The sooner the athletes play, the better. Moreover, the best measure is to start within the bottom line, which can put more pressure on the opponent. After serving, the backhand attacking athletes use the backhand to grab high points from the baseline, which sometimes poses a greater threat to the opponent than hitting hard. That is, it is commonly referred to as turning back. The literature [10] analyzed the impression of female tennis players from physical, technical, and psychological factors, and elaborated on the characteristics and trend distribution of the playing styles in modern female tennis. Moreover, it proposed that today's female tennis players have great offensive skills, and there will be more female players adopting more active offensive styles. For this reason, coaches should focus on developing young tennis players in the most ideal way and be fully prepared for future offensive play. The literature [11] believes that in tennis, players tend to adopt a certain style of play based on their own unique technical, physical, tactical, and psychological characteristics, and imitating a style of play at a young age also has an impact on the type of play used by an athlete. Moreover, it believes that players who want to develop the best type should be players with comprehensive skills. For this reason, coaches and athletes should ensure that training sessions and personal training include five actual combat training items: serving, receiving, bottom counter attack, cross-ball, and online volley. The literature [12] believed that most players play more defensively than offensively during the game. Even so, the result is that the mistakes in the game are greater than the active scoring, and in the game, the risk of offense is greater than that of defense. The literature [13] studied the differences in the tactics used by all-round players in the face of different play types. In the face of serving and Internet players, all-round players often have to improve their first serve success rate; that is, it does not hurt to spin more and slow down when serving. Moreover, by changing the serve point, all-round players can serve the opponent's body more often, thus avoiding the opponent's direct attack when receiving the serve. The literature [14] mentioned that the two-handed backhand stroke technique is the backhand stroke technique used by most athletes. Some athletes who are born with height and strength conditions are not particularly dominant, but have good flexibility and fast speed, and can use the two-handed backhand technique to better develop their tennis potential. At the same time, it recommends that players strengthen their physical fitness while learning to use the two-handed backhand technique, and continuously improve their tennis IQ, so that they can master the technique more quickly and promote the popularization of tennis. The literature [15] divided the technique of receiving and serving into two sections in the research of receiving and serving tennis. Among them, one is the front section of the shot, and the other is the shot section. The front part of the shot is composed of three parts: grip, position, and body posture. The position is generally near the bottom line and close to the sideline of singles, but the position should be selected according to the serving power and spin of the server and one serve and two serve. The literature [16] used the knowledge of system theory to conduct in-depth research on tennis serve when exploring the tennis serve system, and believed that tennis serve is not just a technical action, but a complete system. The literature [17] pointed out that the speed of serving is an effective way to affect the opponent's offensiveness and direct scoring. Under the condition of a certain speed, the player's choice of the ball's landing point can avoid the opponent's special skills and attack the opponent's weakness, which can more effectively restrain the opponent's performance and win the victory of the serve. The literature [18] used the American Peak three-dimensional high-speed video system and the video analysis system of the Aijie Human Information Institute to measure and analyze the kinematics data of the strong serve technique of domestic outstanding female tennis players. In the end, it is concluded that the vigorous serve of tennis is a whipping action. Moreover, the basic principles of the technique of serving are proposed and demonstrated. In addition, it is proposes that the hitting action is not a natural transition and extension of the swing action, but a relatively independent action technique with its own technical characteristics. The literature [19] took high-quality tennis matches 
as the research object. The success rate, scoring rate, straightness rate, and the outcome of a high-quality tennis match are related as follows. Those with a high success rate often cannot win the game, while those with a high scoring rate have a very high probability of winning. The literature [20] concluded that the first serve success rate and the first serve win rate in a tennis match are not positively correlated, and the statistical value of the first serve success rate has no substantial significance, and its level has little effect on the outcome of the game. However, the first serve win rate and the second serve win rate have a greater impact on the outcome of the game, and they are the two key indicators that determine the outcome of the game. From the above analysis, it can be seen that the relevant technology for tennis match analysis is currently relatively vacant. Therefore, this article combines the actual needs of the tennis match to construct a corresponding visual analysis model, and performs action feature recognition and strategy improvement with the support of big data technology.

\section{Machine Learning Model}

This paper proposes three methods of action knowledge extraction based on the random forest model, so the first step is to establish a random forest model. In the process of establishing the random forest model, a data set $(X, Y)$ is given. Among them, $X=\left\{x^{1}, \ldots, x^{N}\right\}$ is the attribute vector set and $Y=\left\{y^{1}, \ldots, y^{N}\right\}$ is the classification label set. Each attribute vector $\vec{x}^{i}=\left\{x_{1}^{i}, \ldots, x_{M}^{l}\right\}$ has $M$ attribute values; among them, each attribute value $x_{j} \in D_{j}$ has a finite value range $D_{j} \cdot x_{j}$ can be categorical or numerical. The classification label $Y$ has a finite value range $D_{Y}$. If there is no ambiguity, $\vec{x}^{i}=\left\{x_{1}^{i}, \ldots, x_{M}^{i}\right\}$ will also be represented by $\vec{x}=\left\{x_{1}, \ldots, x_{M}\right\}$ below. In order to simplify the description, this paper first only considers the case where the prediction classification is binary; that is, $y \in\{0,1\}$. Only small changes are needed to extend the binary classification situation to a multiclass model [21].

A random forest model generally consists of $K$ decision trees. Each decision tree $k$ has an output function $o_{k}(\vec{x})$, which means that a classification mark $y \in D_{y}$ is output when the input attribute vector is given. Therefore, the output of the entire random forest is

$$
H(\vec{x})=\sum_{k=1}^{k} w_{k} o_{k}(\vec{x}) .
$$

Among them, $w_{k} \in R$ is the weight of each decision tree. In addition, $H(\vec{x})$ also has another probability representation. A classification mark $c \in D_{Y}$ is given:

$$
p(y=c \mid \vec{x})=\frac{\sum_{k=1}^{k} w_{k} I\left(o_{k}(\vec{x})=c\right)}{\sum_{k=1}^{k} w_{d}} .
$$

Among them,

$$
I\left(o_{k}(\vec{x})=c\right)
$$

is an indicator function; when $o_{k}(\vec{x})=c$, its value is $l$, otherwise it is 0 . An input vector $\vec{x}$ is given, and the output prediction classification mark $y_{H}$ of the random forest model $H(\vec{x})$ is [22]

$$
y_{H}=\arg \max _{c \in D_{Y}} p(y=c \mid \vec{x})
$$

The random forest model modeling algorithm generally uses the following steps: for $k=1, \ldots, k$,

(1) $n_{k}\left(0<n_{k}<N\right)$ data are sampled from the data set with replacement.

(2) According to the $n_{k}$ data, a decision tree without pruning is trained. When building a decision tree model, the attribute set used to create a branch node is to randomly select a part of all attributes instead of using the entire attribute set to create a branch node. The output of most random forest models adopts a special form of formula (1) that is, we set $w_{k}=1 / k$. Therefore, formula (1) is simplified to [23]

$$
H(\vec{x})=\sum_{k=1}^{k} \frac{1}{k} o_{k}(\vec{x})
$$

Definition 1. Attribute segmentation: a random forest algorithm model $H(\vec{x})$ will be given, and each attribute $x_{i}, i=$ $1, \ldots, M$ will be divided into a certain number of intervals [24]:

(1) If $x_{i}$ is categorized, and it contains $n$ categories, then $x_{i}$ is logically divided into $n$ intervals.

(2) If $x_{i}$ is numerical, if it is assumed that the branch node in each decision tree in the random forest algorithm model is $x_{i}>b$, then $b$ is a dividing point of attribute $x_{l}$. If there are $n$ division points for attribute $x_{l}$ in all decision trees, then attribute $x_{l}$ is divided into $n+1$ intervals.

The number of segmentation intervals of attribute $x_{i}$ is expressed by $n_{i}$, so naturally, the segmentation vector $\vec{p}=$ $\left(p_{1}, \ldots, p_{M}\right)$ corresponds to the attribute vector $\vec{x}=\left(x_{1}, \ldots, x_{M}\right)$. Among them, the value of $\vec{p}$ is the index value of the segmentation interval where the attribute $x_{l}$ is located. Because the attribute values of the $\vec{p}$ vector and the $\vec{x}$ vector correspond to each other, it is convenient for the following description. In the absence of ambiguity, $\vec{x}$ will still be used to represent the segmentation vector $\vec{p}$ below [25].

Definition 2. Attribute change: a random forest algorithm model $H(\vec{x})$ is given, and $\tau$ represents attribute change, which is defined as a triplet $\tau=\left(x_{l}, p, q\right)$. Among them, $x_{i}, i=1, \ldots, M$, is a certain attribute, and the values of $p$ and $q$ represent two of $x_{l}$ attributes. If and only if there is an attribute value $x_{i}$ of the $i$-th attribute in the vector in the interval $P$, the attribute change $\tau$ is executable in the given vector $\vec{x}$. The function of the attribute change $\tau$ is to 
complete the transfer of the attribute value $x_{i}$ of the vector from the interval $p$ to the interval $q$.

Definition 3. Action: a change set of attributes is defined as action $a, a=\left\{\tau_{1}, \ldots, \tau_{|a|}\right\}$, and there is $\tau_{|a|} \geq 1$, which means that at least one attribute change is in one action. The condition that an action executable on the $\vec{x}$ vector needs to meet is that all attribute changes in the action can be executed on the vector $\vec{x}$. The execution of action $a$ will generate a cost value; that is, $\pi(a)>0$.

For the given attribute vector $\vec{x}$ and the action $a$ that can be performed on each attribute of $\vec{x}$, the final result vector of executing action a is $\vec{x} \otimes a$.

In the absence of ambiguity, $p=(y=c \mid \vec{x})$ is simply described as $p(\vec{x})[26]$.

Definition 4. Action knowledge extraction problem: the AKE (actionable knowledge extraction) problem can be represented by a four-tuple $\prod=\left(H, \vec{x}^{I}, c, A\right)$, and the $H$ in the four-tuple represents the random forest algorithm model. At the same time, $\vec{x}$ represents the input vector of the problem, $c \in D_{Y}$ represents the final desired classification label, and $A$ is the action set. Finding an action sequence $A_{s}$ is the ultimate goal of the AKE problem; among them, there is $A_{s}=\left\{a_{1}, \ldots, a_{n}\right\}, a_{l} \in A$. The constraints to be satisfied by the action sequence $A_{s}$ are

$$
\min _{A_{s} \in A} F\left(A_{s}\right)=\sum_{a_{l} \in A_{s}} \pi\left(a_{i}\right) .
$$

Therefore, we obtain

$$
p\left(\vec{x}^{g}\right) \geq z
$$

Among them, $z$ represents a constant, $0<z \leq 1$, and the target vector $\vec{x}^{g}$ is

$$
\vec{x}^{g}=\vec{x}^{I} \otimes a_{1} \otimes \ldots \otimes a_{n}
$$

That is, the action sequence $A_{s}=\left\{a_{1}, \ldots, a_{n}\right\}$ is sequentially applied to the input vector $\vec{x} I^{1}$, and the result vector is finally obtained.

State is an ordered set of a minimum of variables $q_{0}, q_{1}, \ldots, q_{n}$ introduced to describe the difference between a certain type of different things, and its vector form is as follows:

$$
Q=\left[q_{0}, q_{1}, \ldots, q_{n}\right]^{T}
$$

Each element $q_{l}=(i=0,1, \ldots, n)$ in the formula is a component of the set, which is called a state variable. Given a set of values for each component, a specific state is obtained:

$$
Q_{k}=\left[q_{0 k}, q_{1 k}, \ldots, q_{n k}\right]^{T} .
$$

The means of changing a problem from one state to another is called an action, which is generally represented by $a$.

The state space of a problem (state space) is a graph that represents all possible states and their relationships to solve the problem. The state space is generally a directed graph, denoted by $G=(V, A, G)$. Among them, $V$ represents a node set, which is a set of all possible states. $A$ represents the set $\left(a_{1}, a_{2}, \ldots, a_{n}\right)$ of actions related to solving the problem, which can be used to transform one state into another state. $G$ represents the set of all possible goal states related to solving the problem.

The model of Markov decision process is shown in Figure 1, which is generally represented by the five-tuple $\langle S, A, T, R, \gamma\rangle$, where $S$ represents a finite set of all possible states of an agent or environment. Each state in the set represents a possible state, and each state must contain all relevant signs for making correct decisions; $A$ represents a limited set of all possible actions of an agent; $T$ represents the state transition function $T\left(s, a, s^{\prime}\right)$, which is used to express the probability of performing the action $a$ in the state $S$ to reach the state $s^{\prime}$. The Markov property is reflected in the state transition function. The future state of the decision is only related to the current state of the decision and the currently selected action. The above content can be formally expressed as

$$
P\left(s_{t+1}=s^{\prime} \mid s_{t}, a_{t}, s_{t-1}, a_{t-1}, \ldots, s_{0}, a_{0}\right)=P\left(s_{t+1}=s^{\prime} \mid s_{t}, a_{t}\right) \text {. }
$$

$R$ represents the reward function, and $R(s, a)$ represents the immediate reward value that the agent can obtain by performing action $a$ in state $S$. The reward function can be related to the subsequent state, that is, $R\left(s, a, s^{\prime}\right)$. Under this definition, all $s^{\prime}$ of $R\left(s, a, s^{\prime}\right)$ can be expected to be converted into $R(s, a) . \gamma$ is the discount factor, and its value range can be expressed as $\gamma \in[0,1]$. The reason for setting the discount factor is to make the long-term return of the infinite number of steps converge, which makes the Markov decision process model have strong practical significance. When human beings make decisions, most of them pay more attention to the value of returns in a short period of time, thus downplaying the final long-term returns. When the $\gamma$ value is close to 1 , it means that the agent values long-term returns. When $\gamma$ is close to 0 , it means that the agent pays more attention to the immediate report. The Markov decision process model can control the impact of current decisions on the future by setting the size of $\gamma$.

The classic planning problem can be described as a triple form $D=\langle I, A, G\rangle$. Among them, $I$ represents the initial state set, $A$ represents the action set, and $G$ represents the target state set. In this type of problem, the initial state, characteristic action, and target state are all required to be clearly stated. The action that can change the current state of the system is an action, and the action sequence represents the process evolution of the system state, as shown in Figure 2.

\section{Markov Decision Process to Solve the Optimization Problem of Action Knowledge Extraction}

Reinforcement learning learns decision-making strategies through autonomous interaction with the environment, so that the long-term cumulative reward value received by the 


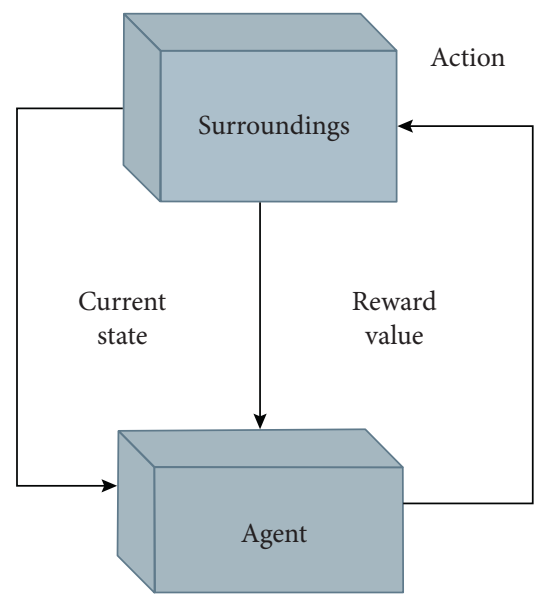

Figure 1: Markov decision process.

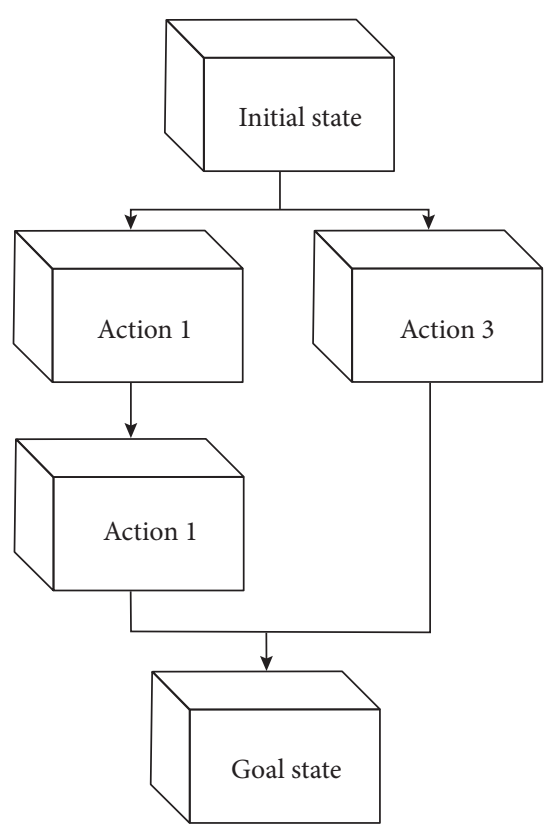

Figure 2: Classical planning problem description.

strategy is the largest. The difference between reinforcement learning and other machine learning methods is that it needs to learn strategies through interaction with the environment. Secondly, after an action is performed in the environment, if there is no mark on the quality of the action, and only after a period of interaction, the accumulated reward can be known, so as to infer the quality of the previous action. This is a forward-looking process. The idea of Markov decisionmaking process is used, and the expected target state can be obtained by determining the change of state attributes through the reward function. Finally, the action knowledge is extracted, and the action knowledge can be transformed into intuitive recommendations for users.

The most basic model of Markov decision process is a five-tuple $\prod_{M D P}=\{S, A, T, R, \gamma\}$; among them, $S$ represents a finite set of states, that is, the state space, and the state is represented by $s ; A$ represents the action space of a limited action set, and the action is represented by $a$; $T: S \times A \times S \longrightarrow[0,1]$, as the state transition probability matrix, represents the probability distribution of transitioning to another state after performing an action in a certain state; $R$ represents the reward function:

$$
R_{s}^{a}=E\left[R_{t+1} \mid S_{t}=s, A_{t}=a\right] .
$$

The above formula represents the immediate reward made by the environment when the state transition occurs; $\gamma$ represents the discount factor, and $\gamma \in[0,1]$. The size of $\gamma$ indicates the degree of inclination to the present and future rewards. The introduction of $\gamma$ is the uncertainty of the future, which can avoid the infinite return of the Markov process. When $\gamma$ is close to 0 , it is more inclined to the immediate reward value of the current time step. When $\gamma$ is close to 1 , it is more inclined to the long-term reward value in the future.

The reward function $R$ is the reward signal obtained during the interaction with the environment. The reward function reflects the nature of the current task and also serves as the basis for strategy modification. The reward function $R$ is to evaluate the quality of the generated action:

$$
R_{s}=E\left[R_{t+1} \mid S_{t}=s\right] G_{t} .
$$

We assume that $G_{t}$ represents the return value of all discount rewards from time step $t$ :

$$
G_{t}=R_{t+1}+\gamma R_{t+2}+\ldots,=\sum_{k=0}^{\infty} \gamma^{k} R_{t+k+1} .
$$

Here, the discount factor is used to control the return value $G_{t}$ to be limited, and $\gamma^{k} R$ is the reward value $R$ obtained through the influence of the $k+1$ time step discount.

For each state $S$ in the state set $S$, the strategy $\pi$ needs to complete an action $a$ in the action set $A$; that is, it specifies the set of actions to be taken in each possible state. Strategy $\pi$ is the mapping from state to action, that is, $\pi: S \longrightarrow A$.

In the Markov decision process, our goal is to find an optimal strategy $\pi^{*}$ with the largest cumulative reward $R_{\pi}$ :

$$
\begin{aligned}
& \pi^{*}=\arg \max _{\pi} R_{\pi}, \\
& R_{\pi}=E_{\pi}\left[\sum_{t=0}^{\infty} \gamma^{t} r_{t}\right] .
\end{aligned}
$$

Among them, $\pi^{*}$ is the optimal strategy, $r_{t}$ is the immediate reward for actions performed at time step $t$, and $R_{\pi}$ is the cumulative reward for actions performed at time step $t$ under strategy $\pi$. At the same time, $\gamma^{t}$ is the discount factor $\gamma \in[0,1]$ to the power of $t$, and $E_{\pi}[\cdot]$ is the expectation under strategy $\pi$.

For any strategy, the expected return of long-term accumulated rewards obtained by executing the strategy is used to evaluate the pros and cons of the strategy. The reward function $R$ is an instant evaluation of a certain state (or action), but the value function considers the quality of a certain state (or state-action pair) from a long-term perspective, so the value function is usually called evaluation function. 
The expectation of accumulative rewards obtained by executing action $a_{t}$ and subsequent strategy $\pi$ in state $s_{t}$ is denoted as $V\left(s_{t}\right)$; that is,

$$
V\left(s_{t}\right)=E\left(\sum_{t=0}^{\infty} \gamma^{t} r_{t+1}\right)
$$

Among them, $r_{t}$ is the immediate reward of the action performed at time $t$, and the discount factor $\gamma(\gamma \in[0,1])$ balances the current immediate reward value with the future reward value and ensures the convergence of $V(s)$.

For any strategy $\pi$, the value function is defined to accumulate the expected value of the discount reward value; that is,

$$
V^{\pi}(s)=E_{\pi}\left[\sum_{t=0}^{\infty} \gamma^{t} r_{t} \mid s_{0}=s\right] .
$$

Among them, $s_{t}$ represents the state of time step $t$, and $t=0$ represents that $s_{0}$ corresponds to the initial state.

The purpose of the reinforcement learning algorithm is to find a strategy $\pi$, which is the ultimate goal of reinforcement learning, so that the value $V^{\pi}(s)$ of each state $S$ reaches the maximum at the same time, namely,

$$
\begin{aligned}
V^{\pi}(s) & =E\left\{r_{1}+\gamma r_{2}+\ldots+\gamma^{i-1} r_{i}+\ldots \mid s_{0}=s\right\}, \\
V^{*}(s) & =\max _{\pi} V^{\pi}(s) .
\end{aligned}
$$

Among them, $r_{t}$ is the immediate reward at time step $t$, and $\gamma(\gamma \in[0,1])$ is the discount factor. $V^{*}(s)$ is called the optimal value function, and the corresponding optimal strategy is

$$
\pi^{*}=\arg \max _{\pi} V^{\pi}(s) .
$$

According to Bellman's optimal equation, there are

$$
\begin{aligned}
V^{*}(s)= & \max _{a} E\left[r_{t+1}+\gamma V^{*}\left(s_{t+1}\right) \mid s_{t}=s, a_{t}=a\right], \\
& =\max _{a} \sum_{s^{\prime}} T\left(s, a, s^{\prime}\right)\left[R(s, a)+\gamma V^{*}\left(s^{\prime}\right)\right] .
\end{aligned}
$$

Among them, $V^{*}\left(s^{\prime}\right)$ is the optimal state value function in the next state $s^{\prime}$.

Algorithm performs strategy iteration: the algorithm first randomly initializes a strategy $\pi_{t}$ and calculates the state value function $v_{t}$ under this strategy. According to these state value functions, a new strategy $\pi_{t+1}$ is obtained, and the value function $v_{t+1}$ of each state under the new strategy is calculated until convergence. Calculating the value of each state under a strategy is called strategy evaluation. A new strategy based on the state value is called strategy improvement.

Algorithm performs strategy evaluation: according to Bellman's equation, the value function of a state is related to the value function of its subsequent states. Therefore, we use the subsequent state value function $v\left(s^{\prime}\right)$ to update the current state value function $v(s)$. Strategy evaluation traverses all states and updates its state value function according to the following formula:

$$
V^{\pi_{t}}(s)=\sum_{a \in A} \pi(s, a)(R(s, a))+\gamma \sum_{s^{\prime}} T_{\left(s, a, s^{\prime}\right)} V^{\pi_{t+1}}\left(s^{\prime}\right) .
$$

Algorithm performs strategy improvement: the new strategy is obtained according to the state value function, and the new strategy is better than the old strategy. For a state $S$, the strategy chooses an action a that maximizes the current state value function $R(s, a)+\gamma \sum_{s^{\prime}} T_{\left(s, a, s^{\prime}\right)} V^{\pi}\left(s^{\prime}\right)$.
That is,

$$
\pi_{t+1}=\left\{\begin{array}{cc}
1 & a=\arg \max _{a}\left(R_{(s, a)}+\gamma \sum_{s^{\prime}} T_{\left(s, a, s^{\prime}\right)} V^{\pi_{t+1}}\left(s^{\prime}\right)\right), \\
0 & a \neq \arg \max _{a}\left(R_{(s, a)}+\gamma \sum_{s^{\prime}} T_{\left(s, a, s^{\prime}\right)} V^{\pi_{t+1}}\left(s^{\prime}\right)\right) .
\end{array}\right.
$$

The termination conditions of the strategy iteration are

$$
V^{\pi_{t}}(s)= \begin{cases}0, & \text { If state } S \text { is the target state, } \\ R_{(s, a)}+\gamma \sum_{s^{\prime}} T_{\left(s, a, s^{\prime}\right)} V^{\pi_{t+1}}\left(s^{\prime}\right), & \text { otherwise. }\end{cases}
$$

Input: $\prod_{A K E}=\{H, \vec{x}, c, A\}$

Output: $a_{t}$

Strategy $\pi(s, a)$, state $s_{t}$, action $a_{t}$, reward $r_{t}$ immediately, and transition probability $T_{\left(s, a, s^{\prime}\right)}$ is initialized.
Repeat

In the current state $s_{t}$, action $a_{t}$ is selected according to the greedy strategy.

Action $a_{t}$ is executed, and the next round of state $s_{t+1}$ and immediate reward $r_{t}$ are obtained. 
The algorithm first performs a strategy evaluation:

Then, the algorithm performs strategy selection:

$$
V^{*}(s)=\sum_{a \in A} \pi(s, a)\left(R_{(s, a)}+\gamma \sum_{s^{\prime}} T_{\left(s, a, s^{\prime}\right)} V^{\pi_{t+1}}\left(s^{\prime}\right)\right) .
$$

$$
\pi_{t+1}=\left\{\begin{array}{cc}
1 & a=\arg \max _{a}\left(R_{(s, a)}+\gamma \sum_{s^{\prime}} T_{\left(s, a, s^{\prime}\right)} V^{\pi_{t+1}}\left(s^{\prime}\right)\right), \\
0 & a \neq \arg \max _{a}\left(R_{(s, a)}+\gamma \sum_{s^{\prime}} T_{\left(s, a, s^{\prime}\right)} V^{\pi_{t+1}}\left(s^{\prime}\right)\right) .
\end{array}\right.
$$

Until the termination condition is met, the algorithm exits:

$$
V^{\pi_{t}}(s)= \begin{cases}0, & \text { If state } S \text { is the target state, } \\ R_{(s, a)}+\gamma \sum_{s^{\prime}} T_{\left(s, a, s^{\prime}\right)} V^{\pi_{t+1}}\left(s^{\prime}\right), & \text { otherwise. }\end{cases}
$$

\section{Tennis Video Image Processing Based on Image Processing}

The research objects and data analysis in this article are all from professional tennis players' matches and training, which are stored in the form of video images. Due to the complicated background of tennis competition and training, it is difficult to identify the characteristics of tennis. This article combines image processing technology to design an automatic processing system for tennis video images. The core of the system is the TS201 processor. This processor has good image signal processing capabilities and is a widely used image processor. The composition of the whole system includes image processor TS201, detector, storage device, RS422 serial port device, and so on. Figure 3 shows the architecture of the image automatic processing system.

The workflow of the tennis video image automatic processing system is as follows: the high-resolution camera collects the image signal; after the image is filtered, it is sent to the image processor TS201 through the RS422 serial port and the signal transmission link; the feature recognition of the signal is completed in the image processor, the feature type is determined, and the image processing result is sent to the memory.

Image processing technology is the core of the tennis video image automatic processing system. The efficiency of image processing determines the efficiency of automatic monitoring. This article focuses on the key aspects of image processing, such as image segmentation, noise filtering, and edge detection.

(1) In the process of image automation processing in this article, the first thing to do is image segmentation. Image segmentation refers to segmenting the more obvious parts of the image according to certain pixel values and image grayscale and other reference factors, and at the same time removing blurry images. The threshold method is usually used to segment the image, and the specific operation steps are divided into the following three steps. The first step is to traverse the pixel values in the image and find the point $M_{0}\left(x_{0}, y_{0}\right)$ with the largest pixel value. The second step is to combine the actual quality of the image, artificially set the size of the image with $4 * 3$ pixels, and compare the maximum pixel point $M_{0}\left(x_{0}, y_{0}\right)$ up, down, left, and right. Pixel compares the size of the found pixel value with the current maximum pixel value and finds the point $M_{1}\left(x_{1}, y_{1}\right)$ with the smallest pixel value.

The third step is to obtain the difference between the maximum pixel value and the minimum pixel value in the image. The formula is as follows:

$$
i=M_{0}\left(x_{0}, y_{0}\right)-M_{1}\left(x_{1}, y_{1}\right) \text {. }
$$

(2) Noise Filtering. The interference signals in the image cannot be completely avoided, so these noise signals must be filtered in a targeted manner. The image signal is

$$
f(x, y)=g(x, y)+\alpha(x, y) .
$$

In the formula, $g(x, y)$ is the characteristic image; $\alpha(x, y)$ is the noise signal in the image.

Commonly used noise filtering methods include Kalman filtering, median filtering, and so on. The gray value of the noise signal in the image is 


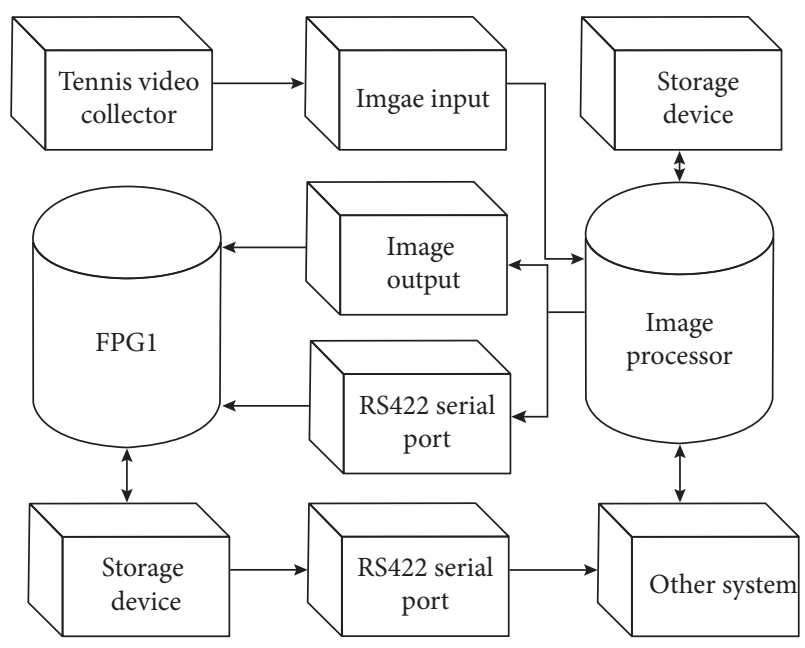

FIgURE 3: Motion monitoring image system architecture.

obviously different from the gray value of the image. Therefore, this paper adopts the median filtering method to filter the signals with abnormal gray value in the image to obtain higher quality images.

(3) Edge Detection. In the process of image processing, edge detection plays a very important role in the recognition of image feature types. Edge detection is also a key step to achieve target recognition. Because the edge image of the target object is obviously different from the background, the feature recognition of the target can be effectively performed by using edge detection technology. The edge detection operators used in this article are Sobel operator and Robert operator during image processing. The function of the image operator is to perform accurate filtering of the edge image, which helps to improve the recognition efficiency of feature types.

\section{Competition Technical Analysis}

This paper first obtains the special objects of tennis match technical analysis through data mining technology and obtains the most representative research objects. After that, this paper specifically analyzes the tennis technology of the research object and names the five research objects $A, B, C$, $D$, and $E$ obtained by data mining in this paper. The basic conditions are shown in Table 1.

In this paper, two professional digital cameras are used to shoot the subject with fixed-point and fixed-focus shooting. Moreover, this paper takes five outstanding professional graduate students with six years or more of tennis experience as the research objects, arranges them to do overhand serve techniques and shoot them on the spot. The shooting frequency is $240 \mathrm{~Hz}$, the shutter speed is $1 / 2000 \mathrm{~s}$, the range of the three-dimensional calibration frame is $2.5 \mathrm{~m}$ in diameter, and the angle between the two cameras and the test object is $90^{\circ}$, as shown in Figure 4 .

In terms of handle, the five subjects usually play continental style. However, each tester's handle style changed during the serve. $A$ and $B$ use the anti-Eastern style, while $C, D$, and $E$ use a handle between the continental style and the anti-Eastern style (see Figure 5 for the specific handle). It can be seen that the five subjects all changed their handling styles when switching from playing to serve, and the " $V$ " in the mouth of the clapping hand changed from the front type to the back type, but each subject had a different degree of conversion.

Because the five test subjects in this study are all righthanded rackets, in order to facilitate the description of the left and right sides of the batter's body in the paper, the "right side" in the text refers to the bat's "holding side" and the "left side" is the bat's "nonholding side."

Throwing the ball vertically helps the hitter avoid too much wrong prediction of the spatial position of the ball, thus greatly improving the accuracy of the shot. When throwing the ball, the athlete should ensure that all joints of the upper arm are fully extended, and the relative positions of the upper arm, shoulder, elbow, and body are directly determined. Moreover, the athlete should throw the ball to the upper right front of the body to provide protection for the accurate hitting of the ball when preparing to hit the ball with the right arm swing. In addition, the forward deviation of the position of the ball relative to the body is to conform to the inertia of the person swinging forward. Therefore, the slight forward shift when throwing the ball is to effectively ensure that the ball can be hit directly above the body when swinging. Table 2 and Figures 6 and 7 show the results of the research the position of throwing shots for the flat strike and the top spin.

From the overall comparison, each subject's throws are different. However, a comparative analysis of each subject's two throwing actions when serving the ball showed that the deviation of the throwing in the two horizontal axes $(X$-axis and $Y$-axis) in the positive direction of the top spin is larger than that of the throwing of the flat strike. In other words, it is more appropriate to throw the ball toward the back left of the batter when a top spin is used.

In the throwing and racket phases, the flexion of the ankle joints, knee and hip joints, and the decrease of the body's center of gravity are sufficient preparations for the explosive force and rapid rebound required for the next vigorous swing. When knees and hips are bent downward, the leg muscles are stretched to a certain extent, which induces stretch and launch, which is conducive to the formation of explosive force. However, the initial length of the musculature varies from person to person, so the optimal angles of the knees and hips that are most conducive to explosive exertion when bending and squatting to lower the center of gravity are also different for different people. Moreover, the results obtained differ among different test subjects. The biggest difference is the smallest joint angle (Table 3 and Figures 8 and 9).

In the racket stage, the movement characteristics of the left and right shoulder joints are mainly reflected in the changes in their spatial positions. In the course of the two kinds of serve techniques, the vertical height difference between the left and right shoulders behaves differently. Among them, the change in the height of the shoulder joint on the nonholding side is that the height of the top spin is higher than that of the flat strike, and the average difference 
TABle 1: Research object information table.

\begin{tabular}{lccccc}
\hline Test object & Age & Height $(\mathrm{m})$ & Weight $(\mathrm{kg})$ & Practice time (years) & Clap hands \\
\hline$A$ & 31 & 1.8 & 68.7 & 8.1 & Right hand \\
$B$ & 32 & 1.8 & 73.7 & 7.1 & Right hand \\
$C$ & 30 & 1.8 & 79.8 & 6.1 & Right hand \\
$D$ & 31 & 1.9 & 82.8 & 8.1 & Right hand \\
$E$ & 29 & 1.8 & 80.8 & Right hand \\
\hline
\end{tabular}

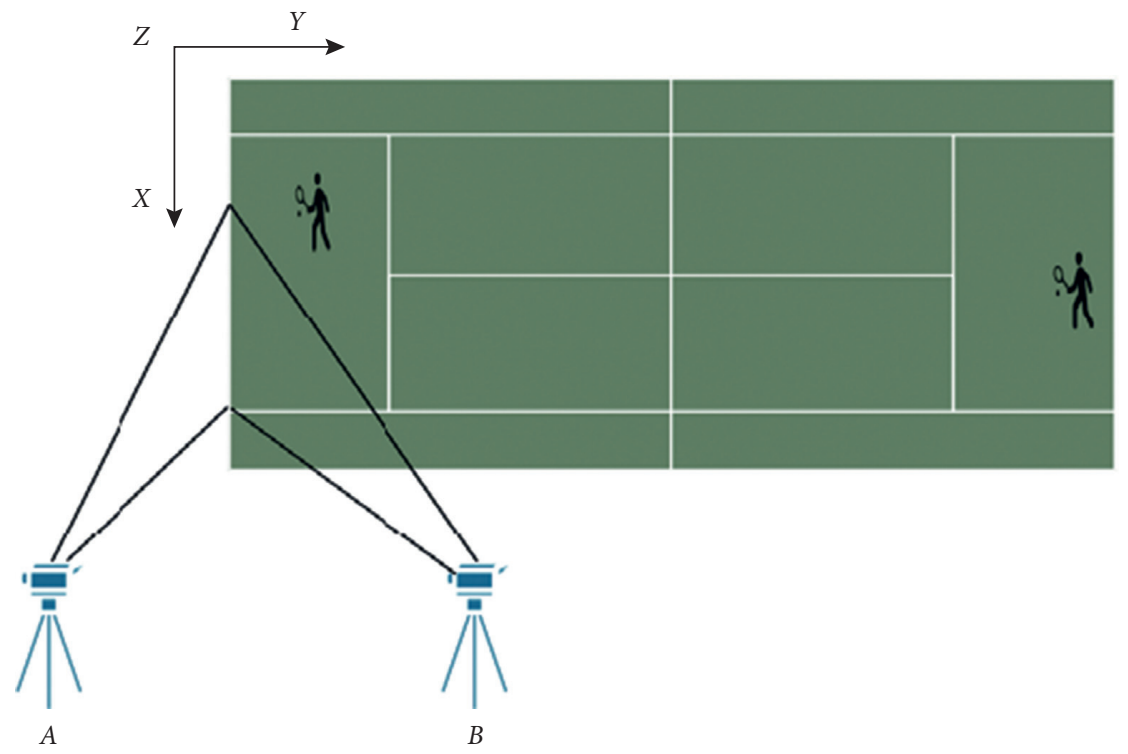

Figure 4: Experimental recording scene.

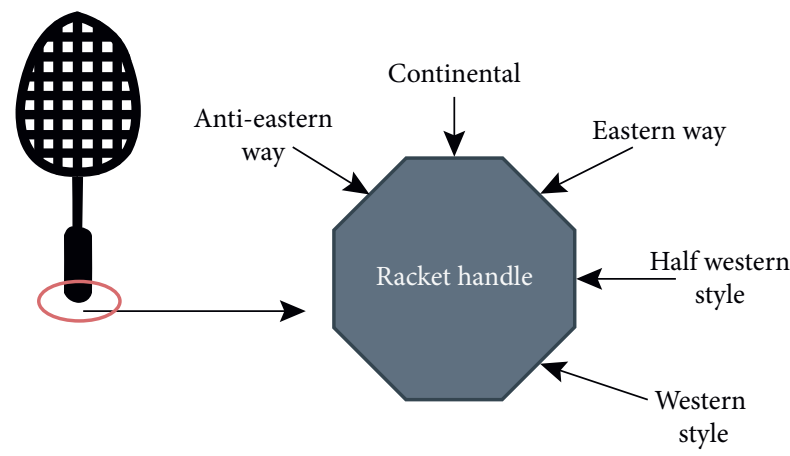

Figure 5: Tennis racket handle.

is three centimeters. However, the change in the vertical height of the shoulder joint on the holding side shows that the height of the top spin is lower than that of the flat strike, and the difference is five centimeters on average. Due to the characteristics of the above two serving techniques, the difference in height between the shoulders is more prominent, and the average difference is about eight centimeters. Similarly, in terms of the level of space between the left and right shoulders, when using top spin, it is also greater than when using flat strike. The difference in the vertical height of the left and right shoulders between the two types of serve is caused by the increase in the degree of squatting of the knee and hip joints on the right side (on the racket side) of the top spin. In this process, the knee and hip joints also have similar characteristics to the shoulder joints. The difference between the knee and hip joints in the two technical actions is reflected in the increase in the top spin.

Table 4 and Figure 10 show the comparison of spatial characteristics at the lowest point of the shoulder joint in the racket stage.

From the motion characteristics of the knee, shoulder, and hip joints, we can conclude that the right knee, hip, and shoulder joints have lower flexion compared to the left knee, hip, and shoulder joints when top spin is used. Moreover, the angles of the right knee and hip joints of the top spin are smaller than the angles of the knee and shoulder joints on the side holding the racket of the flat strike. From this, it can be concluded that in racket action, when the top spin is used, the main joints on the right side are more fully prepared for the explosive power of swing than when the flat strike is used.

From Table 5 and Figure 11, it is found that although the vertical height of the wrist is not very different, the racket and the ground are almost vertical at the moment of hitting when the flat strike is adopted, and the racket is basically tilted flat at the moment of hitting when the top spin is adopted. Therefore, the height of the hitting point is different between the former and the latter.

The height comparison (unit: $\mathrm{m}$ ) of the hitting point of the flat strike and the top spin is shown in Table 6 and Figure 12. 
TABLE 2: Results of the research on the position of throwing shots for the flat strike and the top spin.

\begin{tabular}{lcccccccccc}
\hline Research object & Flat strike & Top spin & Flat strike & Top spin & Flat strike & Top spin & Flat strike & Top spin & Flat strike & Top spin \\
\hline$A$ & 1.67 & 1.77 & 7.39 & 6.88 & 0.67 & 1.08 & -0.94 & -0.21 & 7.09 & 6.83 \\
$B$ & 1.66 & 1.73 & 6.20 & 6.09 & -0.35 & 0.37 & -0.61 & 0.05 & 6.00 & 5.95 \\
$C$ & 1.70 & 1.75 & 6.54 & 6.35 & -0.22 & 0.49 & 0.34 & 0.43 & 6.19 & 6.13 \\
$D$ & 1.69 & 1.75 & 6.33 & 6.22 & -0.41 & 0.25 & 0.55 & 1.26 & 6.39 & 6.30 \\
$E$ & 1.71 & 1.80 & 6.65 & 6.63 & -0.23 & 0.65 & -0.15 & 0.36 & 6.48 & 6.47 \\
\hline
\end{tabular}

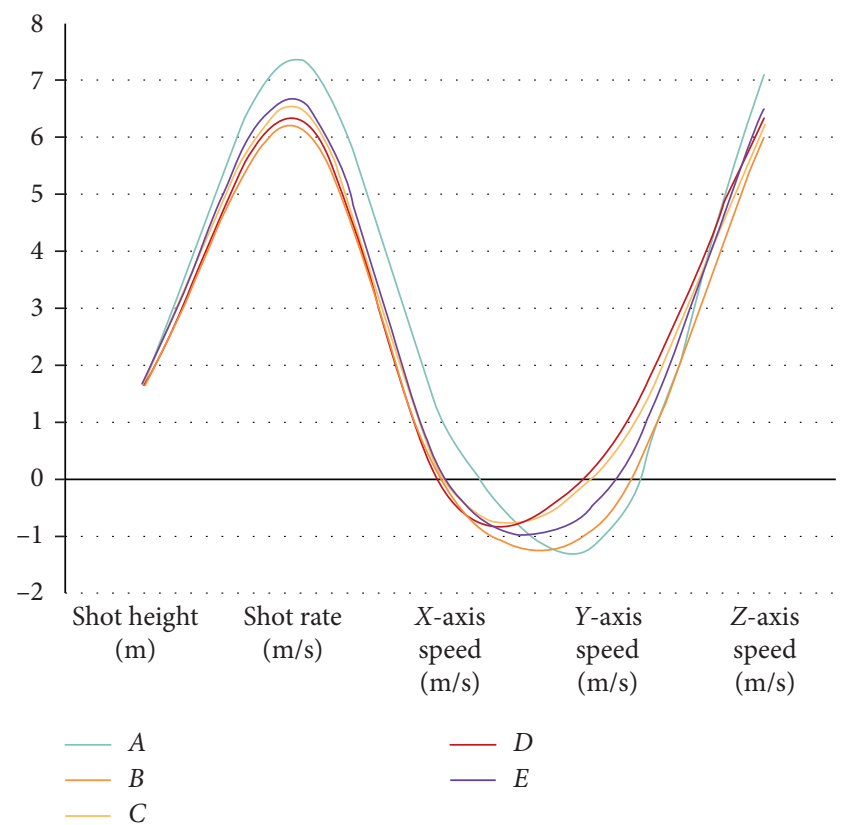

Figure 6: Statistical diagram of the position of the shot of the flat strike.

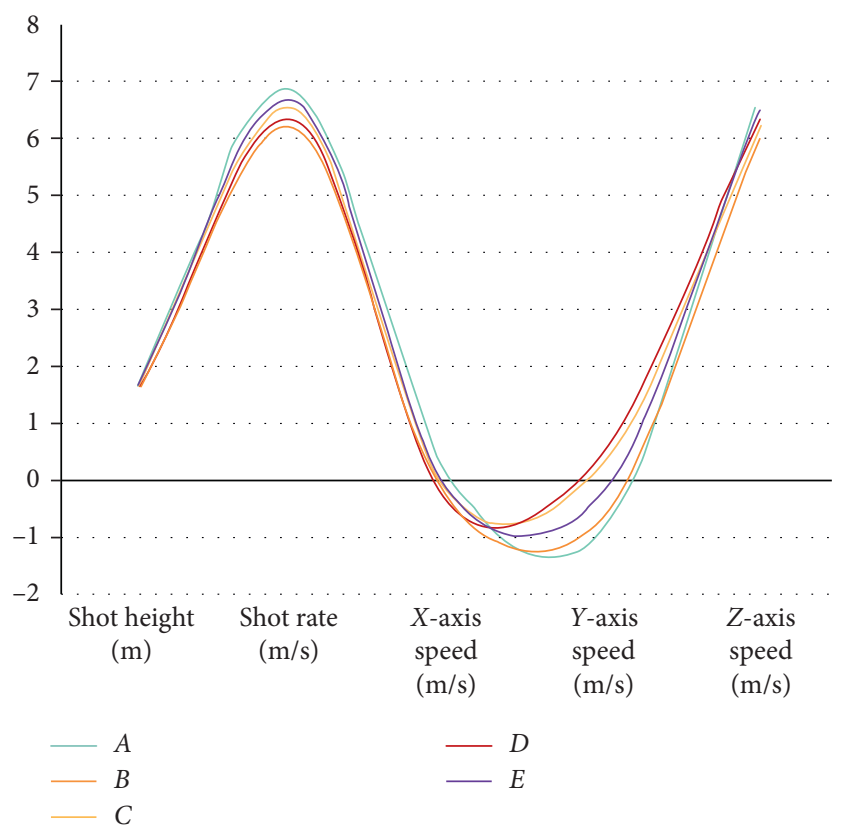

Figure 7: Statistical diagram of the position of the shot of the top spin.
As shown in Table 6 and Figure 12, in tennis, the hitting point refers to the specific spatial position of the ball when it is swung and hitting the ball. In fact, it refers to the relative position of the hitter's body. When the player hits the ball, the spatial position of each part of the hitter also changes, but only the position of the head is relatively fixed. Therefore, it is more practical to regard the position of the head as a relative reference marking point describing the spatial position of the hitting point.

The three-dimensional distance (unit: $\mathrm{m}$ ) between the hitting point and the head space position of flat strike is shown in Table 7 and Figure 13. The three-dimensional distance (unit: $\mathrm{m}$ ) between the hitting point and the head space position of the top spin is shown in Table 8 and Figure 14.

Comparing the two types of serve actions, it can be found that in the top spin, a certain degree of deviation of the ball's spatial position is beneficial to the topspin effect of the ball. However, if the degree of deviation is insufficient, the topspin effect is not obvious. Due to the limitations of various related equipment and other conditions, there is no way to measure the rotation rate of the ball after being hit in the video. However, we can analyze and judge the specific rotation of the ball by using the subspeed of the racket head in other directions at the moment of batting. Through the research and analysis of the video data of the five experimental testers, it can be concluded that the five experimental testers' hitting points when using top spin are all deviated to a certain degree to the left and rear than when using flat strike. Compared with the head space position, the more the hitting point deviates to the left, the higher the speed of the top of the racket at the moment of hitting, and the greater the top spin rate of the ball. Conversely, if the hitting point is shifted to the left insufficiently, the top spin rate of the racket will be relatively reduced at the moment of impact, the top spin rate of the ball will decrease, the lateral spin rate will increase, and the lateral spin rate of the ball will increase. In addition, at the moment before hitting the ball, reasonable elbow bending and wrist swing techniques can have a certain effect on the final speed of the racket head. Moreover, the shaking of the wrist makes a significant difference in the speed of the grip point and the speed of the wrist joint. Because the grip point is close to the wrist joint point, the obvious speed difference must come from the final shaking of the wrist joint. However, the flexion of the elbow and the shaking of the wrist should not be too large. Excessive flexion of the elbow and wrist joints will cause changes in the flight path of the ball and ultimately reduce the quality of the serve. Moreover, it is especially important that a large swipe of the wrist can easily cause the ball to fall into the net. 
TABle 3: Comparison table of smallest angles of the knee and hip joints at the racket stage (unit: ${ }^{\circ}$ ).

\begin{tabular}{cccccccc}
\hline & \multicolumn{2}{c}{ Side knee joint } & \multicolumn{2}{c}{ Right knee joint } & \multicolumn{2}{c}{ Left hip joint } & \multicolumn{2}{c}{ Right hip } \\
& Flat strike & Top spin & Flat strike & Top spin & Flat strike & Top spin & Flat strike \\
\hline$A$ & 85.57 & 89.29 & 78.09 & 74.43 & $A$ & 85.57 & 89.29 \\
$B$ & 92.27 & 94.18 & 110.58 & 102.88 & $B$ & 92.27 & 94.18 \\
$C$ & 76.21 & 82.98 & 103.35 & 104.95 & $C$ & 76.21 & 82.98 \\
$D$ & 100.75 & 102.84 & 92.94 & 85.13 & $D$ & 100.75 & 102.84 \\
$E$ & 92.60 & 86.24 & 111.27 & 104.30 & $E$ & 92.60 & 86.58 \\
\hline
\end{tabular}

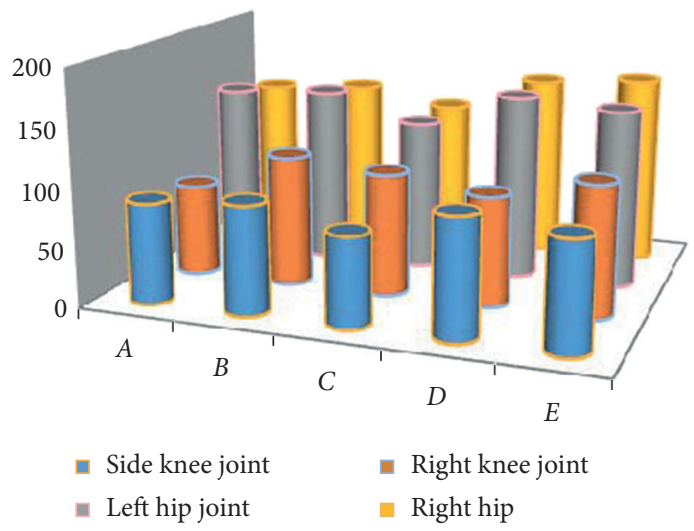

Figure 8: Comparison of the smallest angles of the knee and hip joints at the racket stage of the flat strike.

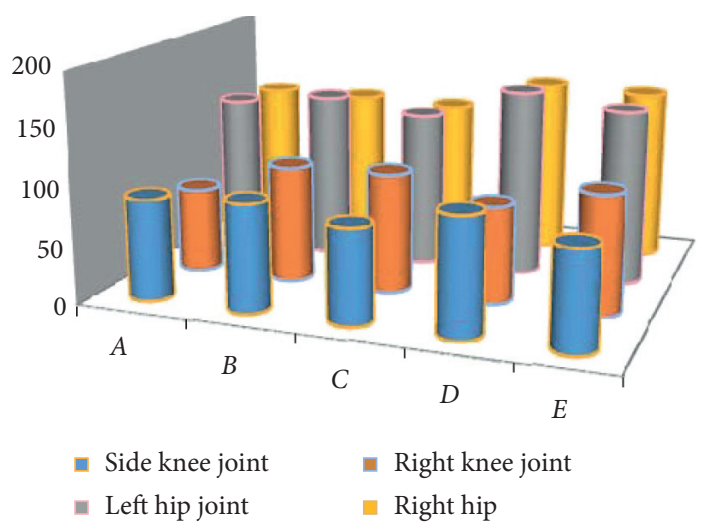

FIgURE 9: Comparison of the smallest angles of the knee and hip joints at the racket stage of the top spin.

TABle 4: Comparison of spatial characteristics at the lowest point of the shoulder joint in racket stage.

\begin{tabular}{lcccc}
\hline & $\begin{array}{c}\text { Left shoulder height } \\
(\mathrm{m})\end{array}$ & $\begin{array}{c}\text { Right shoulder height } \\
(\mathrm{m})\end{array}$ & $\begin{array}{c}\text { Height difference between } \\
\text { shoulders }(\mathrm{m})\end{array}$ & $\begin{array}{c}\text { The angle between the shoulders and the } \\
\text { ground }\left(^{\circ}\right)\end{array}$ \\
\hline Flat strike & 1.31 & 1.16 & 0.15 & 36.76 \\
Top spin & 1.34 & 1.11 & 0.23 & 43.83 \\
Difference & -0.03 & 0.05 & -0.08 & -7.07 \\
\hline
\end{tabular}

In tennis, there are differences in the postures of flat strike and top spin. The specific difference is the angle formed between the flat strike and the top spin. The flat strike is almost perpendicular to the ground at the moment of hitting, while the angle between the top spin and the ground at the moment of hitting is relatively small. By comparing the angles formed between the two serving methods and the ground in Table 9 and Figure 15, it can be 


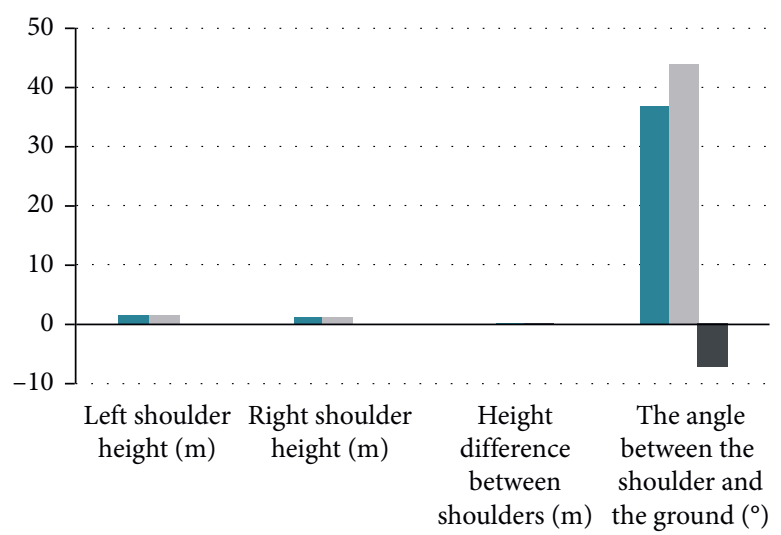

Flat strike

Top spin

Difference

Figure 10: Comparison of spatial characteristics at the lowest point of the shoulder joint in the racket stage.

TABLE 5: Comparison of spatial characteristics at the lowest point of the shoulder joint in racket stage.

\begin{tabular}{lcccccccc}
\hline & Left knee & Right knee & Left hip & Right hip & Left shoulder & Right shoulder & Right elbow & Right wrist \\
\hline Flat strike & 0.74 & 0.77 & 1.07 & 1.13 & 1.46 & 1.76 & 2.06 & 2.27 \\
Top spin & 0.75 & 0.78 & 1.06 & 1.14 & 1.42 & 1.78 & 2.04 \\
Difference & -0.01 & -0.01 & 0.01 & -0.01 & 0.04 & -0.02 & 0.02 & 0.01 \\
\hline
\end{tabular}

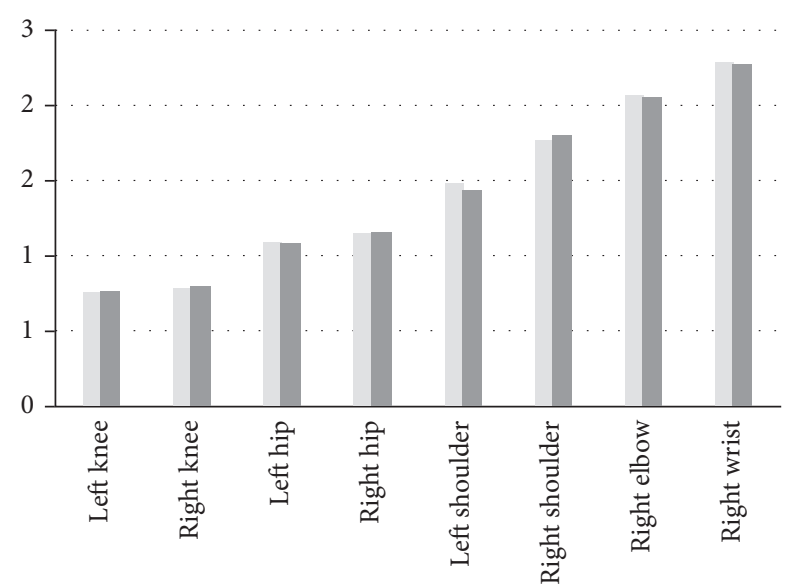

Flat strike

Top spin

FIGURE 11: Comparison of spatial characteristics of the lowest point of the shoulder joint in the racket stage.

TABLE 6: Comparison table of the height of the hitting point of the flat strike and the top spin (unit: $\mathrm{m}$ ).

\begin{tabular}{|c|c|c|c|c|c|c|}
\hline & $A$ & $B$ & $C$ & $D$ & $E$ & Average value \\
\hline Flat strik & 2.69 & 2.67 & 2.86 & 2.67 & 2.76 & 2.73 \\
\hline Top spin & 2.65 & 2.61 & 2.82 & 2.64 & 2.72 & 2.69 \\
\hline Difference & 0.04 & 0.06 & 0.04 & 0.03 & 0.04 & 0.04 \\
\hline
\end{tabular}

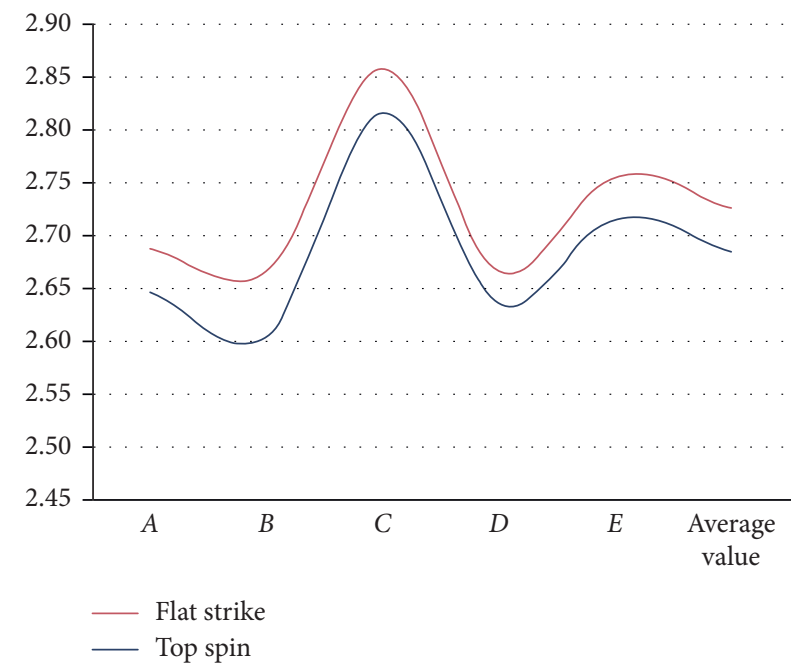

FIGURE 12: Comparison diagram of the height of the hitting point of the flat strike and the top spin (unit: $\mathrm{m}$ ).

TABLE 7: The three-dimensional distance between hitting point and head space position of the flat strike (unit: $\mathrm{m}$ ).

\begin{tabular}{lccc}
\hline & $X$-axis & $Y$-axis & $Z$-axis \\
\hline Hitting point & 1.11 & 0.32 & 1.98 \\
Head position & 1.27 & 0.71 & 1.00 \\
Spatial distance & -0.16 & -0.38 & 0.98 \\
\hline
\end{tabular}




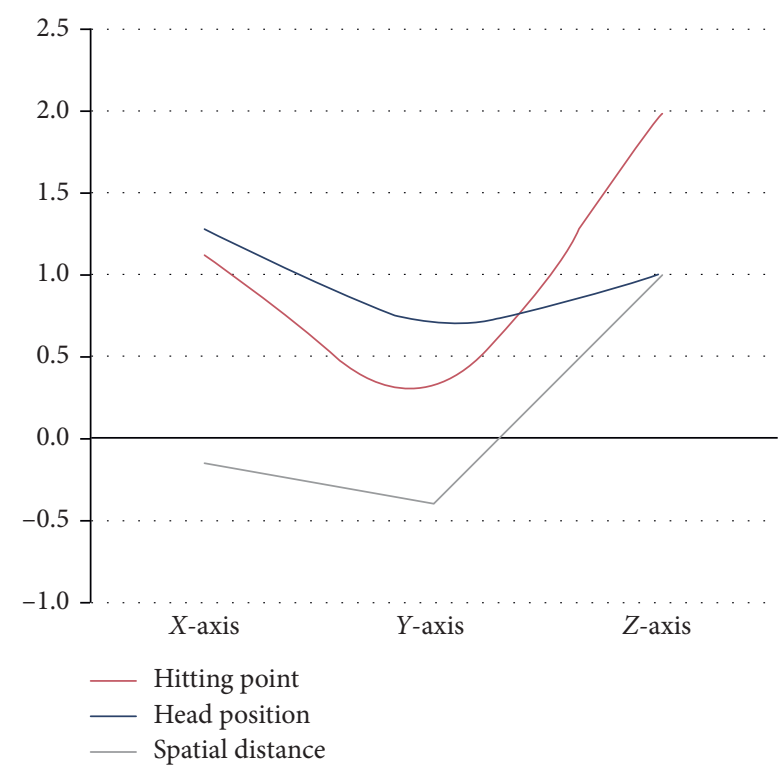

FIGURE 13: Statistical diagram of the three-dimensional distance between the hitting point and the head space position of the flat strike (unit: $\mathrm{m}$ ).

TABLE 8: The three-dimensional distance between hitting point and head space position of the top spin (unit: $\mathrm{m}$ ).

\begin{tabular}{lccc}
\hline & $X$-axis & $Y$-axis & $Z$-axis \\
\hline Hitting point & 1.30 & 0.59 & 1.96 \\
Head position & 1.24 & 0.77 & 0.98 \\
Spatial distance & 0.06 & -0.18 & 0.98 \\
\hline
\end{tabular}

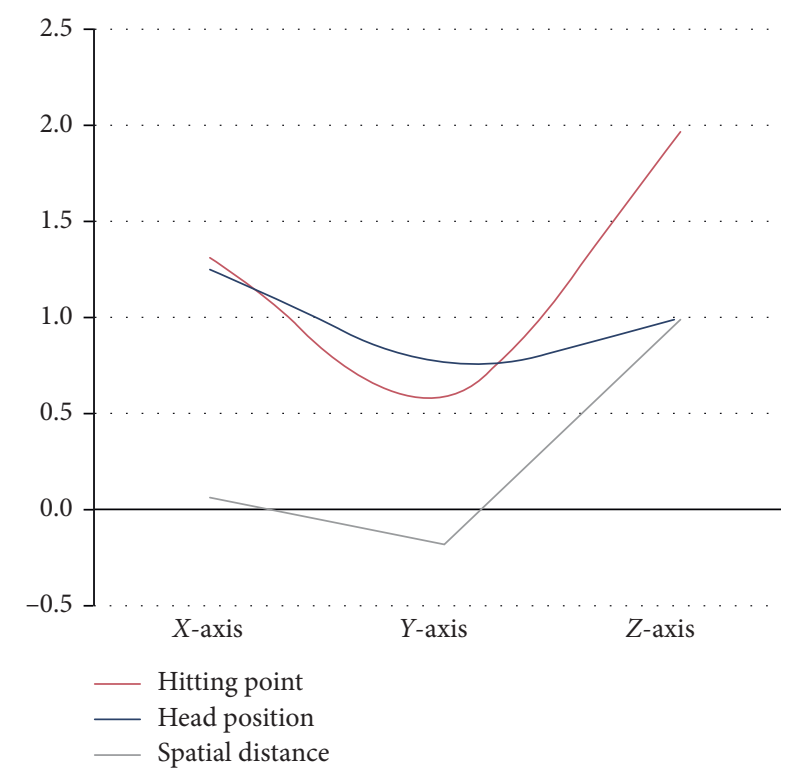

Figure 14: Statistical diagram of the three-dimensional distance between the hitting point and the head space position of the top spin (unit: $\mathrm{m}$ ).

concluded that there is no significant difference in the vertical height of the wrist joints when the two serving methods hit the ball. Therefore, the angle formed between
TABLE 9: Statistical table of the angle between the racket and the ground at the moment of hitting the ball (unit: ${ }^{\circ}$ ).

\begin{tabular}{lcccccc}
\hline & $A$ & $B$ & $C$ & $D$ & $E$ & Average value \\
\hline Flat strike & 70.46 & 71.46 & 71.83 & 67.40 & 60.43 & 68.32 \\
Top spin & 55.37 & 59.24 & 54.28 & 57.96 & 49.92 & 55.36 \\
Difference & 15.09 & 12.22 & 17.55 & 9.43 & 10.50 & 12.96 \\
\hline
\end{tabular}

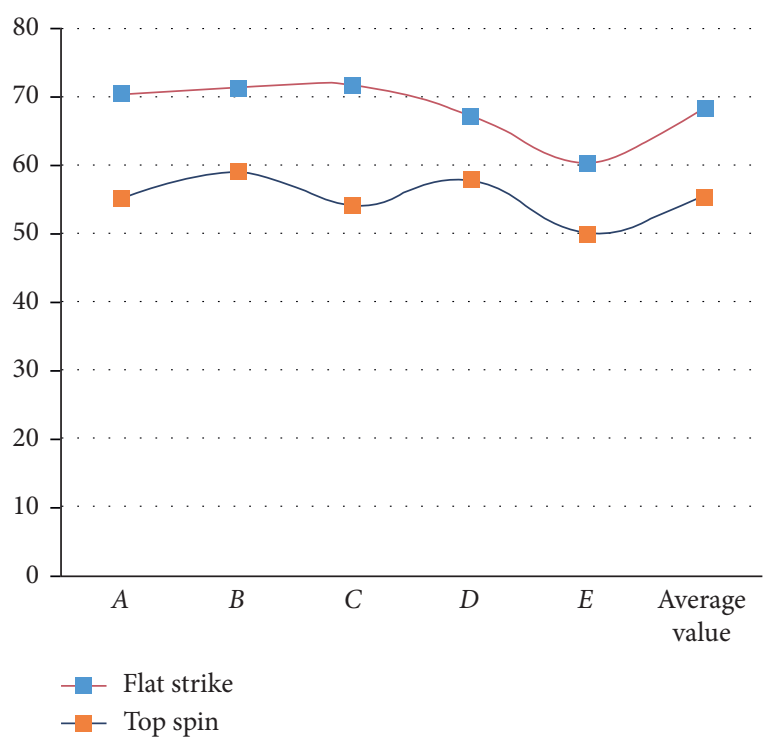

Figure 15: Statistical diagram of the angle between the racket and the ground at the moment of hitting the ball (unit: ${ }^{\circ}$ ).

the moment of hitting and the ground plays a decisive role in determining the vertical height of the hitting point.

Using machine learning to analyze the characteristics of the tennis movement process can effectively improve the effect of feature extraction and discover the problems of athletes in competition and training. Data feature extraction is an effective way to reflect the actual situation through experiments, and it is also an inevitable development of sports direction.

\section{Conclusion}

One of the main focuses of machine learning research is to improve the generalization accuracy and efficiency of model prediction, but in many applications, it lacks the ability to transform prediction results into action knowledge. This paper first introduces the research background and the current research status at home and abroad and gives a concise overview of data mining and knowledge extraction and explains the importance of the study of action knowledge extraction methods from data mining. This paper proposes an action knowledge extraction method that extracts high prediction accuracy and high efficiency decisionmaking action from data and applies this method to the technical analysis of tennis.

With the tremendous advances in data science, the ultimate goal of extracting patterns from data is to facilitate 
decision-making. It is foreseeable that machine learning models will become part of making rational decisions in large-scale AI systems. These modes will be crucial to the support of the action. This research uses SIMI Motion human motion video processing software to display the changing form of each action during the serve in a digital way. Moreover, this research processes the acquired data, analyzes and summarizes the reasonable data obtained by logic and reasoning, and analyzes the data in combination with the model constructed in this paper. The research results show that the performance of the model constructed in this paper is good.

This article uses experimental research methods to extract and analyze the action characteristics of tennis. The model built in this article is only limited to the experimental stage, and the follow-up needs to be studied from the perspective of actual competition and training, and the amount of data in this article is relatively small. In future research, it is needed to use a lot of data for analysis.

\section{Data Availability}

The raw/processed data required to reproduce these findings cannot be shared at this time as the data also form part of an ongoing study.

\section{Conflicts of Interest}

The authors declare that they have no conflicts of interest.

\section{References}

[1] P. S. Glazier and S. Mehdizadeh, "Challenging conventional paradigms in applied sports biomechanics research," Sports Medicine, vol. 49, no. 2, pp. 171-176, 2019.

[2] T. Mullen, J. Highton, and C. Twist, "The internal and external responses to a forward-specific rugby league simulation protocol performed with and without physical contact," International Journal of Sports Physiology and Performance, vol. 10, no. 6, pp. 746-753, 2015.

[3] L. G. Appelbaum and G. Erickson, "Sports vision training: a review of the state-of-the-art in digital training techniques," International Review of Sport and Exercise Psychology, vol. 11, no. 1, pp. 160-189, 2018.

[4] R. Rein and D. Memmert, "Big data and tactical analysis in elite soccer: future challenges and opportunities for sports science," SpringerPlus, vol. 5, no. 1, pp. 1-13, 2016.

[5] K. R. Ridderinkhof and M. Brass, "How kinesthetic motor imagery works: a predictive-processing theory of visualization in sports and motor expertise," Journal of Physiology-Paris, vol. 109, no. 1-3, pp. 53-63, 2015.

[6] D. Mulligan, K. R. Lohse, and N. J. Hodges, "An action-incongruent secondary task modulates prediction accuracy in experienced performers: evidence for motor simulation," Psychological Research, vol. 80, no. 4, pp. 496-509, 2016.

[7] O. M. Khudolii, O. V. Ivashchenko, S. S. Iermakov et al., "Computer simulation of junior gymnasts' training process," Science of Gymnastics Journal, vol. 8, no. 3, pp. 215-228, 2016.

[8] K. A. Tivener and D. S. Gloe, "The effect of high-fidelity cardiopulmonary resuscitation (CPR) simulation on athletic training student knowledge, confidence, emotions, and experiences," Athletic Training Education Journal, vol. 10, no. 2, pp. 103-112, 2015.

[9] O. V. Ivashchenko, O. O. Kapkan, and O. O. Kapkan, "Simulation of process of 14-15 years old girls' training of light athletic and gymnastic exercises," Pedagogics, Psychology, Medical-Biological Problems of Physical Training and Sports, vol. 19, no. 8, pp. 32-39, 2015.

[10] P. D. Owen and N. King, "Competitive balance measures in sports leagues: the effects of variation in season length," Economic Inquiry, vol. 53, no. 1, pp. 731-744, 2015.

[11] J. Yang, "The simulation of table tennis during the course of sports," Caribbean Journal of Science, vol. 52, no. 4, pp. 1561-1564, 2019.

[12] M. Bulat, N. Korkmaz Can, Y. Z. Arslan, and W. Herzog, "Musculoskeletal simulation tools for understanding mechanisms of lower-limb sports injuries," Current Sports Medicine Reports, vol. 18, no. 6, pp. 210-216, 2019.

[13] A. Lopatiev, O. Ivashchenko, O. Khudolii et al., "Systemic approach and mathematical modeling in physical education and sports," Journal of Physical Education and Sport (JPES), vol. 17, no. 1, pp. 146-155, 2017.

[14] N. Bennour, "Teaching practices and student action in physical education classes: perspectives for teacher education," Creative Education, vol. 6, no. 10, pp. 934-944, 2015.

[15] D. Sghaier, M. S. Elandoulsi, and A. Bouassida, "Physical education teacher's training in swimming under the joint didactic action," Creative Education, vol. 6, no. 22, pp. 2433-2437, 2015.

[16] N. Cutforth and E. S. Belansky, "A community-engaged approach to translating research into practice: a physical education story," Progress in Community Health Partnerships: Research, Education, and Action, vol. 9, no. 4, pp. 571-582, 2015.

[17] E.-R. Hwang and T.-Y. Kim, "Intensification of the education of public health, hygiene, and martial arts during the Japanese colonial period (1937-1945)," Journal of Exercise Rehabilitation, vol. 14, no. 2, pp. 160-167, 2018.

[18] D. Murase, K. K. Yokoyama, and Y. Y. YamamotoHasegawa, "Baseball catching patterns differ according to task constraints," Advances in Physical Education, vol. 6, no. 3, pp. 151-157, 2016.

[19] J. T. Spector and M. Lieblich, "Letter regarding "Comparison between low-cost marker-less and high-end marker-based motion capture systems for the computer-aided assessment of working ergonomics" by Patrizi et al. and research reproducibility," Ergonomics, vol. 60, no. 4, pp. 597-598, 2017.

[20] F. Sá, A. Marques, N. B. F. Rocha, M. J. Trigueiro, C. Campos, and J. Schröder, "Kinematic parameters of throwing performance in patients with schizophrenia using a markerless motion capture system," Somatosensory \& Motor Research, vol. 32, no. 2, pp. 77-86, 2015.

[21] M.-K. Kim, T. Y. Kim, and J. Lyou, "Performance improvement of an AHRS for motion capture," Journal of Institute of Control, Robotics and Systems, vol. 21, no. 12, pp. 1167-1172, 2015.

[22] Z.-M. Zhou and Z.-W. Chen, "A survey of motion capture data earning as high dimensional time series," International Journal of Multimedia and Ubiquitous Engineering, vol. 10, no. 9, pp. 17-30, 2015.

[23] M. A. Khan, "Multiresolution coding of motion capture data for real-time multimedia applications," Multimedia Tools \& Applications, vol. 76, no. 15, pp. 1-16, 2016.

[24] A. Puupponen, B. T. Wainio, and T. Jantunen, "Head movements in Finnish Sign Language on the basis of Motion 
Capture data: a study of the form and function of nods, nodding, head thrusts, and head pulls," Sign Language and Linguistics, vol. 18, no. 1, pp. 41-89, 2015.

[25] R. Giannetti, A. Petrella, J. Bach et al., "In vivo bone position measurement using high-frequency ultrasound validated with 3-D optical motion capture systems: a feasibility study," Journal of Medical \& Biological Engineering, vol. 37, no. 7, pp. 1-8, 2017.

[26] H. Zhang, S. L. Wang, S. Chu, S. Chen, H. Meng, and G. Liu, "Application of optical motion capture technology in power safety entitative simulation training system," Optics and Photonics Journal, vol. 6, no. 8, pp. 155-163, 2016. 